\title{
Mini-EUSO: a pathfinder for JEM-EUSO to measure Earth's UV background from the ISS
}

\author{
Marco Ricci* \\ INFN, Laboratori Nazionali di Frascati, Frascati (Roma), Italy \\ E-mail: marco.ricci@lnf.infn.it \\ Marco Casolino \\ INFN, Sezione di Roma Tor Vergata, Roma, Italy, \\ RIKEN Advanced Science Institute, Wako, Japan
}

\section{Pavel Klimov}

Skobeltsyn Institute of Nuclear Physics, Moscow State University, Moscow, Russia

for the JEM-EUSO Collaboration

\begin{abstract}
For any experiment aiming at the observation of Ultra High Energy Cosmic Rays (UHECR) from space, one key measurement is related to the UV background produced in Earth atmosphere. In view of the planned missions (KLYPVE/K-EUSO, JEM-EUSO) at the International Space Station (ISS), a small, compact UV telescope, Mini-EUSO, aiming at the study of the UV night emissions from Earth, is being developed by the JEM-EUSO International Collaboration to be placed at the transparent, nadir looking UV window of the Russian module of the ISS. Mini-EUSO is a mission approved by the Italian Space Agency (ASI) and, under the name "UV atmosphere", by the Russian Space Agency Roscosmos. Scientific, technical and programmatic aspects of this project will be described.
\end{abstract}

The 34th International Cosmic Ray Conference,

30 July- 6 August, 2015

The Hague, The Netherlands

${ }^{*}$ Speaker. 


\section{Introduction}

The measurement and characterization of the UV background produced in the atmosphere of the Earth is a key element for any experiment aiming at the observation of Ultra High Energy Cosmic Rays (UHECR) from space. Such measurements, achievable with small-size, cost-effective devices, besides the intrinsic scientific relevance, can provide useful indications on the design, optimization and performance of full-scale instruments to be placed on board orbiting space stations or free-flyer satellites. Mini-EUSO, aiming at the observation and measurement of the UV night emissions from the Earth, is being developed by the JEM-EUSO Collaboration as a pathfinder and a precursor mission of the planned options for UHECR experiments in space such as KLYPVE/KEUSO [1] on the Russian Segment of the International Space Station (ISS) and JEM-EUSO itself (Extreme Universe Space Observatory on board the Japanese Experiment Module) [2] [3]. Together with other test experiments developed and operated by the JEM-EUSO Collaboration, like EUSO-TA [4] and EUSO-Balloon [5], Mini-EUSO is planned as a next step in the road-map of JEM-EUSO towards the main mission on the ISS. The Mini-EUSO mission originated as a joint project between Italy and Russia, was selected in Italy by the Italian Space Agency (ASI) and is supported by the National Institute of Nuclear Physics (INFN); then, under the name "UV atmosphere", it was approved by the Russian Space Agency Roscosmos and included in the long-term program of space experiments on the ISS. After the signature of a common Scientific Agreement, Mini-EUSO is now an established project between the participating countries of the JEM-EUSO Collaboration.

\section{The Instrument}

The Mini-EUSO instrument, whose scheme and conceptual design are shown in Fig.1, is composed of one single element of the basic JEM-EUSO detection unit, the Photo Detector Module (PDM), consisting of 36 Hamamatsu Multi Anode Photo Multiplier Tubes (MAPMT M64), 64 pixels each, for a total of 2304 pixels. A system of two Fresnel lenses, $25 \mathrm{~cm}$ diameter each, forms the optical system, as described in Sect. 2.1. The front-end electronics is based, opportunely rescaled, on the same ASIC and FPGA boards being developed for JEM-EUSO [6], [7]; the Data Acquisition System and Processing unit is a compact, low-power consumption system as described in Sect. 2.2. Infrared (IR) and Visible (VIS) cameras are foreseen to be installed as ancillary instruments employed to complement the UV observations of the Mini-EUSO focal surface. The compact, Short Wavelength Infrared Camera (SWIR Cam) [8], working as a standalone system, will be attached to the instrument with the main task of performing atmospheric monitoring measurements (as the thermodynamic phase detection of clouds). Data from the cameras will help in the measurements of the emissions of the Earth and the study of transient phenomena.

The Mini-EUSO instrument will be closed in a specific container, a mechanical box providing also all the needed interfaces to the transparent, nadir looking UV window of the Russian module Zvezda of the ISS, as shown in Fig.2.

The main parameters of the instrument, in its flight configuration are listed in Fig.3. 

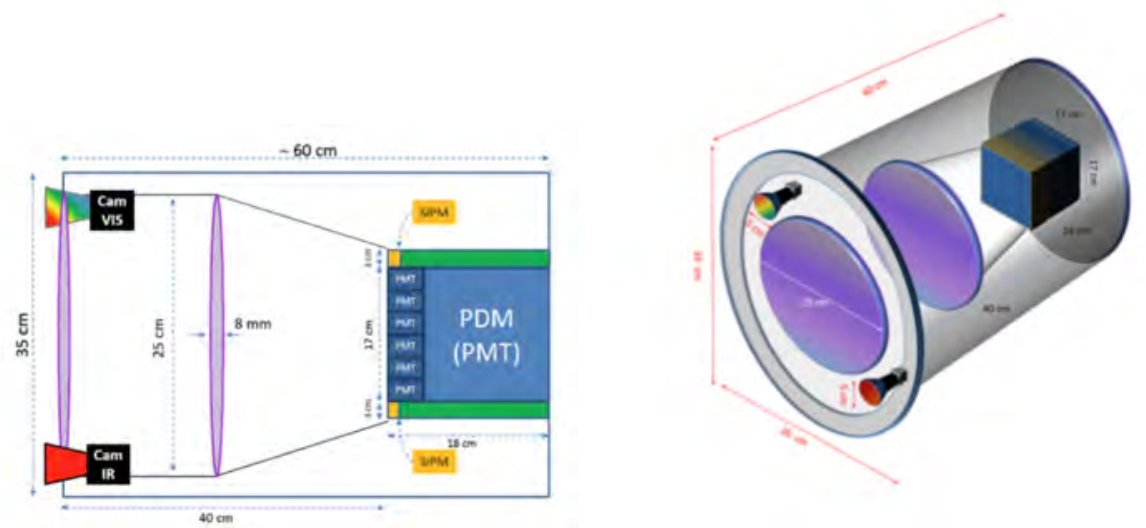

Figure 1: Mini-EUSO scheme and conceptual design.
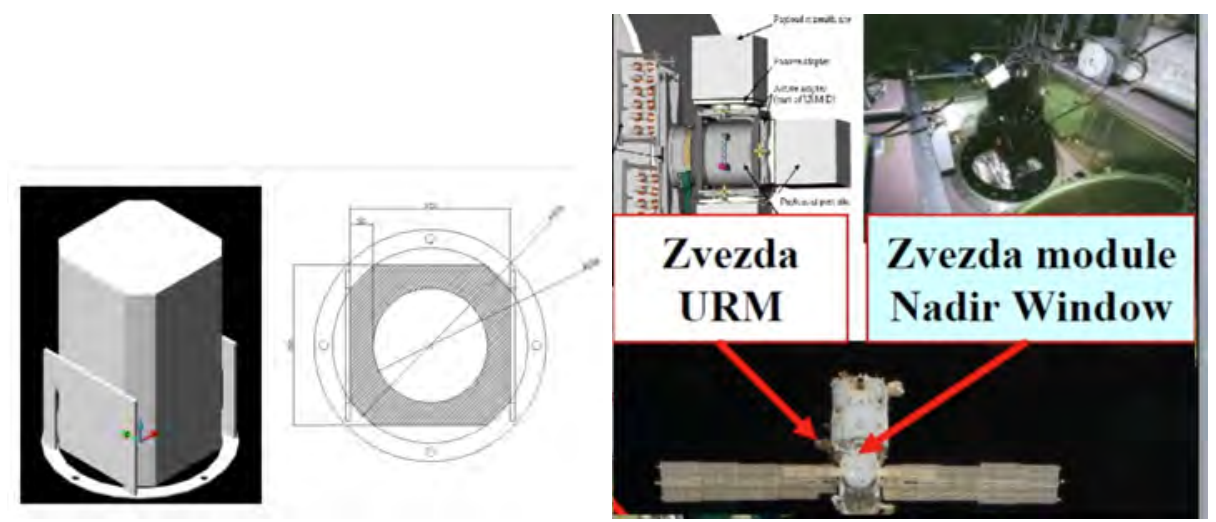

Figure 2: Left: Mechanical box design. Right: Location at Zvezda module UV window.

\begin{tabular}{|l|c|}
\hline \multicolumn{1}{|c|}{ Parameter } & Value \\
\hline Mass, kg & 30 \\
\hline Size, $\mathrm{mm}$ & $350 \times 350 \times 600$ \\
\hline Power, $\mathrm{W}$ & 30 \\
\hline Voltage, $\mathrm{V}$ & 28 \\
\hline Wavelength, $\mathrm{nm}$ & $300-400$ \\
\hline
\end{tabular}

Figure 3: Mini-EUSO instrument main parameters.

\subsection{Optical System}

The optical system designed for Mini-EUSO consists of two double sided flat Fresnel lenses and the focal surface, as shown in Fig. 4. The diameter of both Fresnel lenses is $250 \mathrm{~mm}$. The MiniEUSO optics has a low focal number F\# 1.04, and the effective focal length is $260 \mathrm{~mm}$. Its Field of View (FoV) is $\pm 19^{\circ}$ at $r=900 \mathrm{~mm}$ on the focal surface. The material of the Fresnel lenses is made of UV transparent PMMA (polymethyl methacrylate) [9]. The thickness of the lenses, $5 \mathrm{~mm}$, reduces the mass of the optics resulting in a light system $(\sim 0.3 \mathrm{~kg} / \mathrm{lens})$. The main characteristics of the Fresnel lenses are shown in Table 1, and the point spread function of the optical system is shown in Fig. 5. The photon collection efficiency (PCE), calculated from a raytrace simulation 


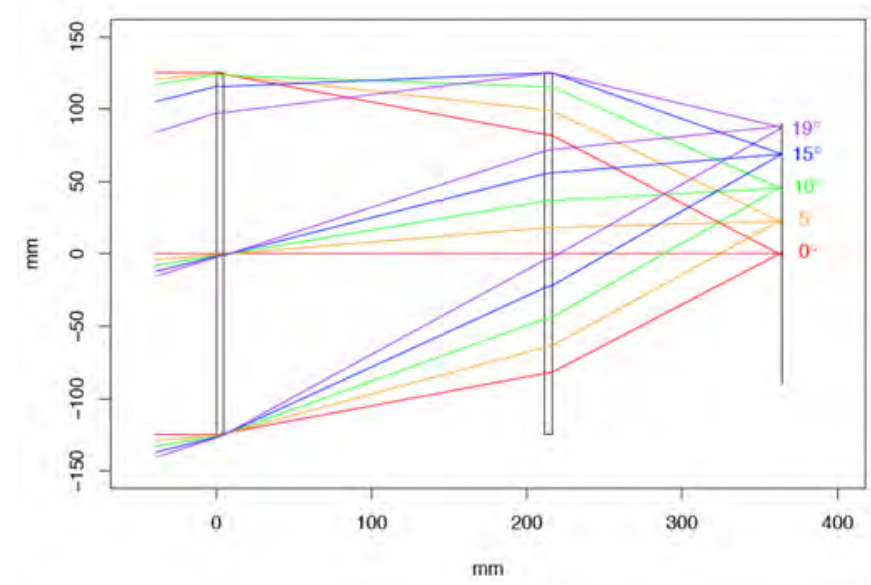

Figure 4: The optical design of the Mini-EUSO telescope.

\begin{tabular}{|l|c|r|r|}
\hline Lens & surface & Groove pitch & Number of grooves \\
\hline \hline \multirow{3}{*}{ Front lens } & Front & $1.91 \sim 21.4 \mathrm{~mm}$ & 33 \\
\cline { 2 - 4 } & Back & $11.75 \sim 56.59 \mathrm{~mm}$ & 5 \\
\hline \multirow{3}{*}{ Rear lens } & Front & $0.01 \sim 32.04 \mathrm{~mm}$ & 11 \\
\cline { 2 - 4 } & Back & $1.63 \sim 21.37 \mathrm{~mm}$ & 36 \\
\hline
\end{tabular}

Table 1: Characteristics of the Fresnel lens surfaces

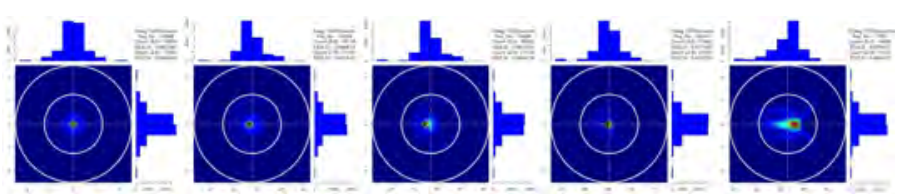

Figure 5: Point spread function of the optical system for light coming parallel with different inclination (from left to right, $0^{\circ}, 5^{\circ}, 10^{\circ}, 15^{\circ}$ and $19^{\circ}$ ). The diameter of the inner white circle is $2.5 \mathrm{~mm}$ and the outer circle is $5 \mathrm{~mm}$.

of the active optical system by a code specifically developed, is $\sim 60 \%$ which takes into account several loss factors as surface reflection, material absorption, surface roughness, Fresnel facet back cut and support structure obscuration.

\subsection{Data Processor and Data Acquisition System}

The Data Processor (DP) is the sub-system of the Mini-EUSO apparatus which performs the control of the instrument and the data management and storage. The main functional requirements of the DP are:

- to configure the front-end electronics

- to manage the mass memory for data storage (removable disks)

- to measure live and dead time of the instrument

- to provide signals for time synchronization of individual events 


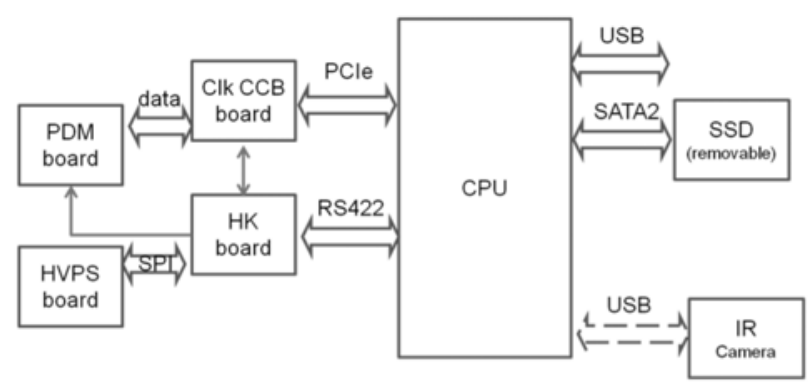

Figure 6: The simplified block diagram of the Mini-EUSO Data Processor.

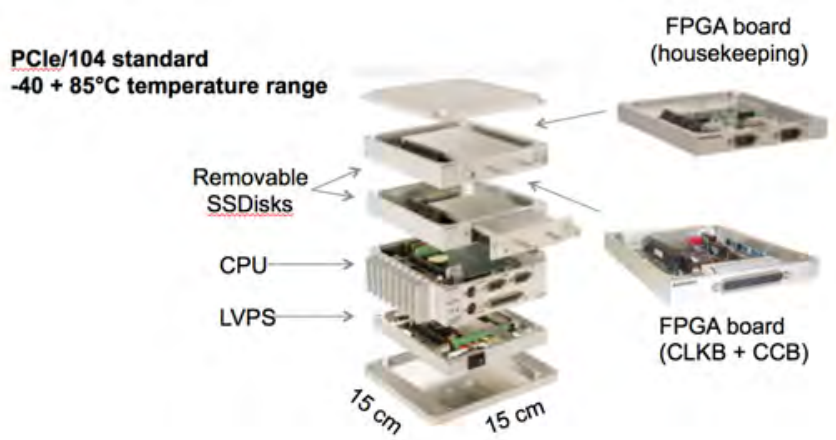

Figure 7: Mini-EUSO Data Handling System.

- to perform housekeeping monitoring

- to control and steer the HVPS (High Voltage Power Supply) system

- to commute from "day" to "night" operating mode and vice versa

- to acquire data from IR and VIS camera

- to provide access to a small part of data which can be transmitted to ground.

The required functionality, an evolution of the DP developed for EUSO-Balloon [10] and EUSO-TA [4], can be obtained by connecting several subsystems performing the various tasks:

- CPU

- Data storage (DST)

- Clock Cluster Control Board (CLK CCBB)

- Housekeeping system (HK)

- Data Processor Power Supply (DP-LVPS)

A simplified block diagram of the DP is shown in Fig. 6.

The requirements on the mass, the power as well as the volume for the DP are $5 \mathrm{~kg}, 15 \mathrm{~W}$ and $(15 \times 15 \times 15) \mathrm{cm}^{3}$ respectively, For the Mini-EUSO DP the PCIe/104 embedded computer standard has been selected. The standard defines both form factors and computer bus, is modular, and allows to stack together boards to produce a customized embedded system. The form factor is somewhat smaller than a desktop PC. The DP is then a stack of six PCIe/104 boards, as shown in Fig. 7. 


\section{Mini-EUSO Science}

The scientific objectives of Mini-EUSO range from cosmic ray to planetary and atmospheric science.

\subsection{Main objective}

Mini-EUSO will be able to provide information of the UV background photon radiance in the wawelength range $300-400 \mathrm{~nm}$. The collected data so far (see Tatiana experiments [11]) give as a reference number $\mathrm{B} \approx(3-9) \cdot 10^{11}$ photons $\cdot \mathrm{m}^{-2} \cdot \mathrm{s}^{-1} \cdot \mathrm{sr}^{-1}$. This value is estimated on the darkest Earth surfaces such as oceans and forests or deserts. However, the value can increase by a factor of $\approx 2$ in presence of clouds, and even by an order of magnitude or more in presence of the moon or for urbanized areas. Balloon experiments, such as NIGHTGLOW [12], BABY [13] and EUSOBalloon [14] provide relevant information on the UV background reflected from ground, but not a direct detection of the airglow emission which is located around $100 \mathrm{~km}$ altitude. Mini-EUSO, with its spatial resolution of $\sim 5 \mathrm{~km}$ and a temporal one of $2.5 \mu \mathrm{s}$ will be able to characterize in a much detailed way the intensity and variation of the UV radiance, airglow included, as a function of time and position of the ISS. These data will be of very much relevance in the proper estimation of the exposure curve of space-based experiments such as JEM-EUSO [15]. Despite its very high energy threshold for cosmic ray detection $\left(E_{t h r} \sim 5 \times 10^{20} \mathrm{eV}\right.$, with its annual exposure of $\sim 15,000 \mathrm{~km}^{2}$ year sr), Mini-EUSO will provide a significant contribution in estimating an absolute limit on the cosmic ray flux above those energies for a null detection. Fig. 8 shows the expected light curve and track on the PDM of an Extreme Energy Cosmic Ray with energy $E=10^{21} \mathrm{eV}$.

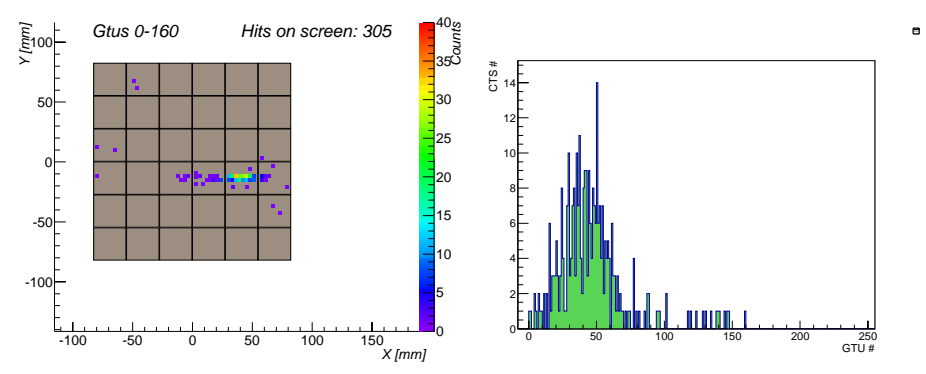

Figure 8: Left: Number of counts recorded in Mini-EUSO by simulating a $10^{21} \mathrm{eV}$ cosmic ray event with 80 degrees inclination from nadir axis. Right: Light curve produced by such a shower. In both plots the UV background light is not added.

\subsection{Additional objectives}

Meteor and fireball observations are key to the derivation of both the inventory and physical characterization of small solar system bodies orbiting in the vicinity of the Earth. For several decades, observation of these phenomena has only been possible via ground-based instruments. Mini-EUSO has the potential to become one of the first operational space-based platforms to share this capability. In comparison to the observation of extremely energetic cosmic ray events, meteor phenomena are very slow, since their typical speeds are of the order of a few tens of $\mathrm{km} / \mathrm{sec}$. By 
scaling the JEM-EUSO performance in meteor detection [16], Mini-EUSO will be sensitive to meteors with magnitudes $\mathrm{M}<+3$.

The observing strategy developed to detect meteors may also be applied to the detection of nuclearites [17], which have higher velocities, a wider range of possible trajectories, but move well below the speed of light and can therefore be considered as slow events as well. The possible detection of nuclearites greatly enhances the scientific rationale behind the Mini-EUSO mission. Preliminary estimations of the sensitivity of Mini-EUSO detection of nuclearites are under study.

Discovered in the late eighties, Transient Luminous Events (TLE), such as red sprites, elves and blue jets are still poorly known. These phenomena, occurring in the upper atmosphere, have been widely studied in recent years. Previous satellite missions (Tatiana-1, Tatiana-2 [11], RELEC [18] ) indicate a high luminosity in UV wavelengths and high frequency of TLEs (especially weak ones).This may affect UHECR measurements and must be carefully studied before the main mission.

Another relevant issue would be the observation of space debris. In more than 50 years of space flight, over 30,000 t of satellites and rockets have been sent to space. It is estimated that almost $3000 \mathrm{t}$ [19] of non-functioning space debris remain in Low Earth Orbit (LEO) in varied forms ranging from fragments to switched off rocket bodies and fully intact multi-ton satellites. Since their orbital velocities are very high, collisions can involve relative impact velocities of the order of $10 \mathrm{~km} / \mathrm{s}$, even the fragments with greater than MJ kinetic energies may cause a severe or catastrophic damage on functioning satellites such as the ISS. As described in [20] Mini-EUSO could be used as a prototype system for tracking such space debris. A super-wide field-of-view telescope (such as JEM-EUSO) and a novel high efficiency fibre-based laser system (CAN) could constitute a very useful orbiting debris remediation system.

Other scientific objectives of Mini-EUSO include the bio-luminescence observation above the sea from space and atmospheric science by coupling UV measurement together with simultaneously taken IR and VIS images.

\section{Technological objectives}

Besides the previously described scientific objectives, Mini-EUSO addresses some important issues by the technological point of view that are summarized as follows:

- First use of Fresnel lenses in Space (meant at an altitude higher than the top of atmosphere $\sim 40 \mathrm{~km}$ ).

- Optimization and validation of JEM-EUSO observational scheme.

- Increase of the Technological Readiness Level (TRL) of JEM-EUSO instrumentation, a typical parameter in the development of devices to be qualified and certified for space.

- Test and R\&D of advanced solutions for future space missions, such as studies on development of SiPM (Silicon Photomultiplier) based photosensors for space applications [21].

Acknowledgment: This work was partially supported by NASA award 11-APRA-0058 in the USA, by Basic Science Interdisciplinary Research Projects of RIKEN and JSPS KAKENHI Grant (22340063, 23340081, and 24244042), 
by the Italian Ministry of Foreign Affairs, General Direction for the Cultural Promotion and Cooperation, by the 'Helmholtz Alliance for Astroparticle Physics HAP' funded by the Initiative and Networking Fund of the Helmholtz Association, Germany, and by Slovak Academy of Sciences MVTS JEM-EUSO as well as VEGA grant agency project 2/0076/13. Russia is supported by the Russian Foundation for Basic Research Grant No 13-02-12175-ofi-m and by Grant No 15-35-21038-mol-a-ved. The Spanish Consortium involved in the JEM-EUSO Space Mission is funded by MICINN \& MINECO under the Space Program projects: AYA2009-06037-E/AYA, AYA-ESP2010-19082, AYA-ESP2011-29489C03, AYA-ESP2012-39115-C03, AYA-ESP2013-47816-C4, MINECO/FEDER-UNAH13-4E-2741, CSD2009-00064 (Consolider MULTIDARK) and by Comunidad de Madrid (CAM) under projects S2009/ESP-1496 \& S2013/ICE-2822.

\section{References}

[1] M. Panasyuk et al. - JEM-EUSO Collaboration, Proc. 34th ICRC, 2015, these proceedings

[2] P. Picozza et al. - JEM-EUSO Collaboration, Proc. 34th ICRC, 2015, these proceedings

[3] A. Olinto et al. - JEM-EUSO Collaboration, Proc. 34th ICRC, 2015, these proceedings

[4] M. Casolino et al. - JEM-EUSO Collaboration, Proc. 34th ICRC, 2015, these proceedings

[5] P. Von Ballmoos et al. - JEM-EUSO Collaboration, Proc. 34th ICRC, 2015, these proceedings

[6] S. Ahmad et al. "SPACIROC: A Front-End Readout ASIC for Spatial Cosmic Ray Observatory", Proc. 32th ICRC, 2011, vol. 3, p. 56

[7] H. Miyamoto et al. "Performance of a front-end ASIC for JEm-EUSO", Proc. 32th ICRC, 2011, vol. 3 , p. 152

[8] E. Joven et al. "Proposal of a Near Infrared Camera for Mini-EUSO", IAC Technology Division DE/CD-JEU/028, May 11, 2015 - Instituto de Astrofisica de Canarias

[9] Y. Takizawa et al. Proc. 33rd ICRC, Rio de Janeiro 2013

[10] G. Osteria et al. - JEM-EUSO Collaboration, Proc. 34th ICRC, 2015, these proceedings

[11] G.K. Garipov et al., Astropart. Phys., 24 (2005) 400.

[12] L.M. Barbier et al., Astropart. Phys., 22 (2005) 439.

[13] O. Catalano et al., Nucl. Instr. Methods A, 480(2-3) (2002) 547.

[14] M. Bertaina et al. - JEM-EUSO Collaboration, Proc. 34th ICRC, 2015, these proceedings

[15] J.H. Adams Jr. et al. - JEM-EUSO Coll., Astrop. Phys. 44 (2013) 76

[16] M. Bertaina et al. - JEM-EUSO Coll., Experimental Astronomy (2014), DOI $10.1007 / \mathrm{s} 10686-014-9375-4$

[17] A. De Rujula and S.L. Glashow, Nature 312 (1984) 734.

[18] S. Svertilov, Proc. 40th COSPAR Scientific Assembly, Moscow 2014 vol. 40, p. 3246

[19] J.-C. Liou and P. N. Krisko, Proc. 64th Int. Astronautical Congress (Beijing), IAC-13-A6.4.2 (2013).

[20] T. Ebisuzaki et al., Acta Astronautica 112 (2015) 102.

[21] A. Haungs et al. - JEM-EUSO Collaboration, Proc. 34th ICRC, 2015, these proceedings 